\title{
ON THE ADDITIVE FORMULAE OF THE THETA FUNCTIONS AND A COLLECTION OF LAMBERT SERIES PERTAINING TO THE MODULAR EQUATIONS OF DEGREE 5
}

\author{
LI-CHIEN SHEN
}

\begin{abstract}
We examine the connection between the additive formulae of the theta functions, the Fourier series expansion of the 12 elliptic functions, and the logarithmic derivatives of the theta functions. As an application, we study the Lambert series related to the modular equations of degree 5 and many interesting identities of Ramanujan are found in this process.
\end{abstract}

\section{INTRODUCTION}

The following two identities play a pivotal role in the study of the modular equations of degree 5 :

$$
\begin{aligned}
& \vartheta_{2}^{2}(0 \mid \tau)-\vartheta_{2}^{2}(0 \mid 5 \tau)=4 q^{\frac{1}{2}} \prod_{n=1}^{\infty}\left(1+q^{2 n}\right)\left(1+q^{5 n}\right)\left(1-q^{5 n}\right)^{2}\left(1+q^{10 n-5}\right), \\
& \vartheta_{3}^{2}(0 \mid \tau)-\vartheta_{3}^{2}(0 \mid 5 \tau)=4 q \prod_{n=1}^{\infty}\left(1+q^{2 n-1}\right)\left(1+q^{5 n}\right)\left(1-q^{5 n}\right)^{2}\left(1+q^{10 n}\right) .
\end{aligned}
$$

The main purpose of this paper is to systematically reexamine the above identities as well as a family of identities of this type within the framework of the well-known additive formulae of the elliptic functions. It will be apparent to the reader that the additive formulae which we are about to derive can be used to obtain unlimited number of identities; however, they are particularly suited for the study of the Lambert series related to the modulus equations of degree 3 and degree 5 . In this work we will focus on the degree 5 case only and a collection of 44 identities are obtained in this manner. Among them are the two above-mentioned identities (see (3.1) and (3.3)). Others include several remarkable identities of Ramanujan (for example, see (3.20) and (3.43)).

Before we begin we should say a few words about our notation. We essentially adopt the notation used in Chapters 21 and 22 of Modern Analysis by Whittaker and Watson [5]. In this section, for brevity, we use $\vartheta_{i}(z)$ and $\vartheta_{i}$ to denote, respectively, $\vartheta_{i}(z \mid \tau)$ and $\vartheta_{i}(0), i=1,2,3,4$. When confusion does arise

Received by the editors August 9, 1993 and, in revised form, October 1, 1993.

1991 Mathematics Subject Classification. Primary 33D10; Secondary 11B65.

Key words and phrases. Theta functions, imaginary transformation, Lambert series, Jacobian elliptic functions. 
such as in the identity $\left(1.3 \mathrm{a}^{\prime}\right)$ and in Section 3, the formal notation $\vartheta_{i}(z \mid \tau)$ will be employed. Also the reader's fluency in the basic properties of the theta functions and the theory of elliptic functions is assumed.

We begin our discussion by introducing the following two identities:

$$
\begin{gathered}
\vartheta_{1}(x) \vartheta_{1}(z) \vartheta_{2}(y) \vartheta_{2}(x+y+z)+\vartheta_{1}(y) \vartheta_{1}(x+y+z) \vartheta_{2}(x) \vartheta_{2}(z) \\
=\vartheta_{2} \vartheta_{1}(y+z) \vartheta_{1}(x+y) \vartheta_{2}(x+z)
\end{gathered}
$$

and

$$
\left(\frac{\vartheta_{4}^{\prime}}{\vartheta_{4}}\right)^{\prime}(z)=\vartheta_{3}^{2} \vartheta_{4}^{2} \frac{\vartheta_{3}^{2}(z)}{\vartheta_{4}^{2}(z)}-\vartheta_{3}^{4} \frac{E}{K} .
$$

The identity (1.2a) is listed and proved in $[5$, p. 518] as

$$
d n^{2} u=\frac{d}{d u}\left(\frac{\boldsymbol{\Theta}^{\prime}}{\boldsymbol{\Theta}}(u)\right)+\frac{E}{K},
$$

where $K$ and $E$ are the complete elliptic integrals of the first and second kinds, respectively. The identity (1.1) is of great significance and we shall make some comments on it at the end of this paper.

We will use these two identities to derive a collection of companion identities. To this end, we first consider (1.2a). Replacing $z$ by $z+\pi / 2, z+\pi / 2+$ $\pi \tau / 2, z+\pi \tau / 2$ and using the basic properties of the theta functions, we obtain, respectively,

$$
\begin{aligned}
& \left(\frac{\vartheta_{3}^{\prime}}{\vartheta_{3}}\right)^{\prime}(z)=\vartheta_{3}^{2} \vartheta_{4}^{2} \frac{\vartheta_{4}^{2}(z)}{\vartheta_{3}^{2}(z)}-\vartheta_{3}^{4} \frac{E}{K}, \\
& \left(\frac{\vartheta_{2}^{\prime}}{\vartheta_{2}}\right)^{\prime}(z)=-\vartheta_{3}^{2} \vartheta_{4}^{2} \frac{\vartheta_{1}^{2}(z)}{\vartheta_{2}^{2}(z)}-\vartheta_{3}^{4} \frac{E}{K}, \\
& \left(\frac{\vartheta_{1}^{\prime}}{\vartheta_{1}}\right)^{\prime}(z)=-\vartheta_{3}^{2} \vartheta_{4}^{2} \frac{\vartheta_{2}^{2}(z)}{\vartheta_{1}^{2}(z)}-\vartheta_{3}^{4} \frac{E}{K}
\end{aligned}
$$

Choosing $z=-(x+y) / 2$ in (1.1), we have

$$
\begin{aligned}
\vartheta_{1}(x) \vartheta_{2}(y)-\vartheta_{1}(y) \vartheta_{2}(x) & =\frac{\vartheta_{2} \vartheta_{1}(x+y) \vartheta_{1}\left(\frac{x-y}{2}\right) \vartheta_{2}\left(\frac{x-y}{2}\right)}{\vartheta_{1}\left(\frac{x+y}{2}\right) \vartheta_{2}\left(\frac{x+y}{2}\right)}, \\
\vartheta_{1}(x) \vartheta(y)+\vartheta_{1}(y) \vartheta_{2}(x) & =\frac{\vartheta_{2} \vartheta_{1}(x-y) \vartheta_{1}\left(\frac{x+y}{2}\right) \vartheta_{2}\left(\frac{x+y}{2}\right)}{\vartheta_{1}\left(\frac{x-y}{2}\right) \vartheta_{2}\left(\frac{x-y}{2}\right)} .
\end{aligned}
$$

The second identity of (1.3a) is obtained from the first one with the replacement of $y$ by $-y$. Now multiplying the above two identities together yields

$$
\vartheta_{1}^{2}(x) \vartheta_{2}^{2}(y)-\vartheta_{1}^{2}(y) \vartheta_{2}^{2}(x)=\vartheta_{2}^{2} \vartheta_{1}(x-y) \vartheta_{1}(x+y) .
$$

It is worthwhile to point out here that if we apply the triple product identities for the theta functions to the right-hand side of the first identity of (1.3a), it becomes

$$
\left(1.3 \mathrm{a}^{\prime}\right) \quad \vartheta_{1}(x \mid \tau) \vartheta_{2}(y \mid \tau)-\vartheta_{1}(y \mid \tau) \vartheta_{2}(x \mid \tau)=2 \vartheta_{1}(x-y \mid 2 \tau) \vartheta_{4}(x+y \mid 2 \tau) .
$$


Other companion identities of (1.3a) and (1.4a) will be given later. To keep us in proper focus, we now go directly to the most essential aspects of our method. To this end, we will have to recall the Fourier series expansions of the Jacobian elliptic functions (see [5, pp. 511-512] for the complete listing of these 12 elliptic functions). For the present purpose, which is immediately evident, we will use

$$
\operatorname{csu}=\frac{\pi}{2 K} \cot x-\frac{2 \pi}{K} \sum_{n=1}^{\infty} \frac{q^{2 n} \sin 2 n x}{1+q^{2 n}} \quad\left(u=\frac{2 K x}{\pi}\right)
$$

to illustrate our method. We first note that in terms of the theta function notation, the above identity is equivalent to

$$
\vartheta_{3} \vartheta_{4} \frac{\vartheta_{2}(x)}{\vartheta_{1}(x)}=\cot x-4 \sum_{n=1}^{\infty} \frac{q^{2 n} \sin 2 n x}{1+q^{2 n}}
$$

From (1.5) and (1.3a), we deduce that

$$
\begin{aligned}
(\cot x & \pm \cot y)-4 \sum_{n=1}^{\infty} \frac{q^{2 n}(\sin 2 n x \pm \sin 2 n y)}{1+q^{2 n}} \\
& =\vartheta_{3} \vartheta_{4}\left(\frac{\vartheta_{2}(x)}{\vartheta_{1}(x)} \pm \frac{\vartheta_{2}(y)}{\vartheta_{1}(y)}\right) \\
& =\vartheta_{2} \vartheta_{3} \vartheta_{4} \frac{\vartheta_{1}(x \mp y \quad) \vartheta_{1}\left(\frac{x \pm y}{2}\right) \vartheta_{2}\left(\frac{x \pm y}{2}\right)}{\vartheta_{2}(x) \vartheta_{1}( \pm y) \vartheta_{1}\left(\frac{x \mp y}{2}\right) \vartheta_{2}\left(\frac{x \mp y}{2}\right)} .
\end{aligned}
$$

And recall that (see $[5$, p. 489])

$$
\left(\frac{\vartheta_{1}^{\prime}}{\vartheta_{1}}\right)(x)=\cot x+4 \sum_{n=1}^{\infty} \frac{q^{2 n} \sin 2 n x}{1-q^{2 n}} .
$$

Combining this with (1.4a) and (1.6), we have

$$
\begin{aligned}
\csc ^{2} y & -\csc ^{2} x+8 \sum_{n=1}^{\infty} \frac{n q^{2 n}(\cos 2 n x-\cos 2 n y)}{1-q^{2 n}} \\
= & \left(\frac{\vartheta_{1}^{\prime}}{\vartheta_{1}}\right)^{\prime}(x)-\left(\frac{\vartheta_{1}^{\prime}}{\vartheta_{1}}\right)^{\prime}(y) \\
= & \frac{\left(\vartheta_{1}^{\prime}\right)^{2} \vartheta_{1}(x-y) \vartheta_{1}(x+y)}{\vartheta_{1}^{2}(x) \vartheta_{1}^{2}(y)} \quad\left(\vartheta_{1}^{\prime}=\vartheta_{2} \vartheta_{3} \vartheta_{4}\right) \\
= & \vartheta_{3}^{2} \vartheta_{4}^{2}\left(\frac{\vartheta_{2}(x)}{\vartheta_{1}(x)}+\frac{\vartheta_{2}(y)}{\vartheta_{1}(y)}\right)\left(\frac{\vartheta_{2}(y)}{\vartheta_{1}(y)}-\frac{\vartheta_{2}(x)}{\vartheta_{1}(x)}\right) \\
= & \left(\cot x+\cot y-4 \sum_{n=1}^{\infty} \frac{q^{2 n}(\sin 2 n x+\sin 2 n y)}{1+q^{2 n}}\right) \\
& \cdot\left(\cot y-\cot x+4 \sum_{n=1}^{\infty} \frac{q^{2 n}(\sin 2 n x-\sin 2 n y)}{1+q^{2 n}}\right) .
\end{aligned}
$$

The chief reason that the identities (1.6) and (1.7) are very interesting is due primarily to the fact that one side of the identity is an infinite sum while the other 
side is an infinite product. So by choosing $x, y$, and the variable $q\left(=e^{\pi i \tau}\right)$ appropriately, we can obtain many strikingly interesting identities. For example, the identities (3.1) and (3.2) are obtained from (1.6) and the identities (3.11) and (3.12) are immediate consequences of (1.7). A more detailed discussion of these identities will be given in the next section. For the time being, we will see how to derive the remaining 15 companion identities of (1.3a) and (1.4a).

First applying the familiar imaginary transformation of Jacobi (see [5, p. 474]) to (1.3a), we obtain

$$
\vartheta_{1}(x) \vartheta_{4}(y) \pm \vartheta_{1}(y) \vartheta_{4}(x)=\frac{\vartheta_{4} \vartheta_{1}(x \mp y) \vartheta_{1}\left(\frac{x \pm y}{2}\right) \vartheta_{4}\left(\frac{x \pm y}{2}\right)}{\vartheta_{1}\left(\frac{x \mp y}{2}\right) \vartheta_{4}\left(\frac{x \mp y}{2}\right)} .
$$

And, from $(1.3 b)$ we have

$$
\vartheta_{1}^{2}(x) \vartheta_{4}^{2}(y)-\vartheta_{1}^{2}(y) \vartheta_{4}^{2}(x)=\vartheta_{4}^{2} \vartheta_{1}(x-y) \vartheta_{1}(x+y) .
$$

We now replace $q$ by $-q$ in (1.3b) and (1.4b). Then

$$
\vartheta_{1}(x) \vartheta_{3}(y) \pm \vartheta_{1}(y) \vartheta_{3}(x)=\frac{\vartheta_{3} \vartheta_{1}(x \mp y) \vartheta_{1}\left(\frac{x \pm y}{2}\right) \vartheta_{3}\left(\frac{x \pm y}{2}\right)}{\vartheta_{1}\left(\frac{x \mp y}{2}\right) \vartheta_{3}\left(\frac{x \mp y}{2}\right)}
$$

and

$$
\vartheta_{1}^{2}(x) \vartheta_{3}^{2}(y)-\vartheta_{1}^{2}(y) \vartheta_{3}^{2}(x)=\vartheta_{3}^{2} \vartheta_{1}(x-y) \vartheta_{1}(x+y) .
$$

It is interesting to observe that

$$
\frac{\vartheta_{i}^{2}(y)}{\vartheta_{1}^{2}(y)}-\frac{\vartheta_{i}^{2}(x)}{\vartheta_{1}^{2}(x)}=\frac{\vartheta_{i}^{2} \vartheta_{1}(x-y) \vartheta_{1}(x+y)}{\vartheta_{1}^{2}(x) \vartheta_{1}^{2}(y)}, \quad i=2,3,4,
$$

and

$$
\begin{aligned}
\left(\frac{\vartheta_{1}^{\prime}}{\vartheta_{1}}\right)^{\prime}(x)-\left(\frac{\vartheta_{1}^{\prime}}{\vartheta_{1}}\right)^{\prime}(y) & =\vartheta_{3}^{2} \vartheta_{4}^{2}\left(\frac{\vartheta_{2}^{2}(y)}{\vartheta_{1}^{2}(y)}-\frac{\vartheta_{2}^{2}(x)}{\vartheta_{1}^{2}(x)}\right) \\
& =\vartheta_{2}^{2} \vartheta_{4}^{2}\left(\frac{\vartheta_{3}^{2}(y)}{\vartheta_{1}^{2}(y)}-\frac{\vartheta_{3}^{2}(x)}{\vartheta_{1}^{2}(x)}\right) \\
& =\vartheta_{2}^{2} \vartheta_{3}^{2}\left(\frac{\vartheta_{4}^{2}(y)}{\vartheta_{1}^{2}(y)}-\frac{\vartheta_{4}^{2}(x)}{\vartheta_{1}^{2}(x)}\right) .
\end{aligned}
$$

We now go to the table of identities in Section 3. We see that, from (1.9),

$$
(3.11)=(3.1) \times(3.2)=(3.3) \times(3.4)=(3.7) \times(3.8) .
$$

In particular, from $(3.11)=(3.1) \times(3.2)$, we have the following interesting identity:

$$
\begin{aligned}
& \left\{\sum_{n=1}^{\infty} \frac{q^{n}-q^{3 n}-q^{7 n}+q^{9 n}}{1+q^{10 n}}\right\}\left\{1+\sum_{n=1}^{\infty} \frac{q^{n}+q^{3 n}+q^{7 n}+q^{9 n}}{1+q^{10 n}}\right\} \\
& \quad=\sum_{n=1}^{\infty} \frac{n\left(q^{n}-q^{3 n}-q^{7 n}+q^{9 n}\right)}{1-q^{10 n}} .
\end{aligned}
$$


To obtain the remaining identities, we replace $x$ by $x+\pi \tau / 2$ and $y$ by $y+\pi \tau / 2$ in (1.3a), $x$ by $x+\pi \tau / 2$ and $y$ by $y+\pi \tau / 2$ in (1.3b), and $x$ by $x+\pi / 2$ and $y$ by $y+\pi / 2$ in (1.3c):

$$
\vartheta_{4}(x) \vartheta_{3}(y)+\vartheta_{4}(y) \vartheta_{3}(x)=\frac{\vartheta_{2} \vartheta_{1}(x-y) \vartheta_{3}\left(\frac{x+y}{2}\right) \vartheta_{4}\left(\frac{x+y}{2}\right)}{\vartheta_{1}\left(\frac{x-y}{2}\right) \vartheta_{2}\left(\frac{x-y}{2}\right)},
$$

$$
\vartheta_{4}(x) \vartheta_{3}(y)-\vartheta_{4}(y) \vartheta_{3}(x)=\frac{\vartheta_{2} \vartheta_{1}(x+y) \vartheta_{1}\left(\frac{x-y}{2}\right) \vartheta_{2}\left(\frac{x-y}{2}\right)}{\vartheta_{3}\left(\frac{x+y}{2}\right) \vartheta_{4}\left(\frac{x+y}{2}\right)}
$$

$$
\begin{gathered}
\vartheta_{3}^{2}(y) \vartheta_{4}^{2}(x)-\vartheta_{4}^{2}(y) \vartheta_{3}^{2}(x)=\vartheta_{2}^{2} \vartheta_{1}(x-y) \vartheta_{1}(x+y), \\
\vartheta_{3}(x) \vartheta_{2}(y)+\vartheta_{2}(x) \vartheta_{3}(y)=\frac{\vartheta_{4} \vartheta_{1}(x-y) \vartheta_{2}\left(\frac{x+y}{2}\right) \vartheta_{3}\left(\frac{x+y}{2}\right)}{\vartheta_{1}\left(\frac{x-y}{2}\right) \vartheta_{4}\left(\frac{x-y}{2}\right)}
\end{gathered}
$$

$$
\vartheta_{3}(x) \vartheta_{2}(y)-\vartheta_{2}(x) \vartheta_{3}(y)=\frac{\vartheta_{4} \vartheta_{1}(x+y) \vartheta_{1}\left(\frac{x-y}{2}\right) \vartheta_{4}\left(\frac{x-y}{2}\right)}{\vartheta_{2}\left(\frac{x+y}{2}\right) \vartheta_{3}\left(\frac{x+y}{2}\right)},
$$

$$
\begin{gathered}
\vartheta_{3}^{2}(x) \vartheta_{2}^{2}(y)-\vartheta_{2}^{2}(x) \vartheta_{3}^{2}(y)=\vartheta_{4}^{2} \vartheta_{1}(x-y) \vartheta_{1}(x+y), \\
\vartheta_{4}(x) \vartheta_{2}(y)+\vartheta_{4}(y) \vartheta_{2}(x)=\frac{\vartheta_{3} \vartheta_{1}(x-y) \vartheta_{2}\left(\frac{x+y}{2}\right) \vartheta_{4}\left(\frac{x+y}{2}\right)}{\vartheta_{1}\left(\frac{x-y}{2}\right) \vartheta_{3}\left(\frac{x-y}{2}\right)}, \\
\vartheta_{4}(x) \vartheta_{2}(y)-\vartheta_{4}(y) \vartheta_{2}(x)=\frac{\vartheta_{3} \vartheta_{1}(x+y) \vartheta_{1}\left(\frac{x-y}{2}\right) \vartheta_{3}\left(\frac{x-y}{2}\right)}{\vartheta_{2}\left(\frac{x+y}{2}\right) \vartheta_{4}\left(\frac{x+y}{2}\right)},
\end{gathered}
$$

The identities of the type (1.4a)-(1.4f) are often called the addition formulae for the theta functions and were known to Jacobi. For more identities of this type, see [5, pp. 487, 488, Examples 1, 2, and 3]. A different method of proving these identities is given in [5, p. 467].

Now with these identities in our disposal, we can easily derive identities of the types (1.6) and (1.7). We consider, for example,

$$
d n u=\frac{\pi}{2 K}+\frac{2 \pi}{K} \sum_{n=1}^{\infty} \frac{q^{n} \cos 2 n x}{1+q^{2 n}} .
$$


In terms of the theta functions, it is the same as

$$
\vartheta_{3} \vartheta_{4} \frac{\vartheta_{3}(x)}{\vartheta_{4}(x)}=1+4 \sum_{n=1}^{\infty} \frac{q^{n} \cos 2 n x}{1+q^{2 n}}
$$

So, from (1.3d), we have

(1.11)

$$
\begin{aligned}
2\left(1+2 \sum_{n=1}^{\infty} \frac{q^{n}(\cos 2 n x+\cos 2 n y)}{1+q^{2 n}}\right) & =\vartheta_{3} \vartheta_{4}\left(\frac{\vartheta_{3}(x)}{\vartheta_{4}(x)}+\frac{\vartheta_{3}(y)}{\vartheta_{4}(y)}\right) \\
& =\frac{\vartheta_{1}^{\prime} \vartheta_{1}(x-y) \vartheta_{3}\left(\frac{x+y}{2}\right) \vartheta_{4}\left(\frac{x+y}{2}\right)}{\vartheta_{4}(x) \vartheta_{4}(y) \vartheta_{1}\left(\frac{x-y}{2}\right) \vartheta_{2}\left(\frac{x-y}{2}\right)}
\end{aligned}
$$

and

$$
\begin{aligned}
4 \sum_{n=1}^{\infty} \frac{q^{n}(\cos 2 n x-\cos 2 n y)}{1+q^{2 n}} & =\vartheta_{3} \vartheta_{4}\left(\frac{\vartheta_{3}(x)}{\vartheta_{4}(x)}-\frac{\vartheta_{3}(y)}{\vartheta_{4}(y)}\right) \\
& =-\frac{\vartheta_{1}^{\prime} \vartheta_{1}(x+y) \vartheta_{1}\left(\frac{x-y}{2}\right) \vartheta_{2}\left(\frac{x-y}{2}\right)}{\vartheta_{4}(x) \vartheta_{4}(y) \vartheta_{3}\left(\frac{x+y}{2}\right) \vartheta_{4}\left(\frac{x+y}{2}\right)}
\end{aligned}
$$

Also recall that $[5$, p. 489$]$

$$
\left(\frac{\vartheta_{4}^{\prime}}{\vartheta_{4}}\right)(x)=4 \sum_{n=1}^{\infty} \frac{q^{n} \sin 2 n x}{1-q^{2 n}}
$$

So, from (1.12), and (1.2a) and (1.4d), we deduce that

$$
\begin{aligned}
\left(\frac{\vartheta_{4}^{\prime}}{\vartheta_{4}}\right)^{\prime}(x)-\left(\frac{\vartheta_{4}^{\prime}}{\vartheta_{4}}\right)^{\prime}(y) & =8 \sum_{n=1}^{\infty} \frac{n q^{n}(\cos 2 n x-\cos 2 n y)}{1-q^{2 n}} \\
& =\vartheta_{3}^{2} \vartheta_{4}^{2}\left(\frac{\vartheta_{3}^{2}(x)}{\vartheta_{4}^{2}(x)}-\frac{\vartheta_{3}^{2}(y)}{\vartheta_{4}^{2}(y)}\right) \\
& =-\frac{\left(\vartheta_{1}^{\prime}\right)^{2} \vartheta_{1}(x+y) \vartheta_{1}(x-y)}{\vartheta_{4}^{2}(x) \vartheta_{4}^{2}(y)}
\end{aligned}
$$

And from (1.11) and (1.13), it yields

$$
\begin{aligned}
(1+ & \left.2 \sum_{n=1}^{\infty} \frac{q^{n}(\cos 2 n x+\cos 2 n y)}{1+q^{2 n}}\right)\left(\sum_{n=1}^{\infty} \frac{q^{n}(\cos 2 n x-\cos 2 n y)}{1+q^{2 n}}\right) \\
& =\sum_{n=1}^{\infty} \frac{n q^{n}(\cos 2 n x-\cos 2 n y)}{1-q^{2 n}} .
\end{aligned}
$$


The identity (1.13) is particularly significant. Because by choosing $x=$ $3 \pi \tau / 4, y=\pi \tau / 4$ and replacing $q$ by $q^{\frac{5}{2}}$, it gives the following remarkable identity of Ramanujan:

$$
\sum_{n=1}^{\infty} \frac{n\left(q^{n}-q^{2 n}-q^{3 n}+q^{4 n}\right)}{1-q^{5 n}}=q \prod_{n=1}^{\infty} \frac{\left(1-q^{5 n}\right)^{5}}{1-q^{n}},
$$

which in turn yields one of the most celebrated identities of Ramanujan:

$$
\sum_{n=1}^{\infty} p(5 n+4) q^{n}=5 \prod_{n=1}^{\infty} \frac{\left(1-q^{5 n}\right)^{5}}{\left(1-q^{n}\right)^{6}} .
$$

Two other identities of this nature, which are derived from (1.11), are discussed in Section 4.

\section{PROOFS FOR SOME IDENTITIES}

In this section, we will use the formulae derived in the previous section to establish a collection of identities tabulated in Section 3. Since all the proofs are essentially identical, we will pick out identities (3.1) and (3.6) to illustrate our method. We treat (3.1) first. We observe that choosing $x=0$ in (1.10), we obtain

$$
\vartheta_{3}^{2}=\vartheta_{3}^{2}(0 \mid \tau)=1+4 \sum_{n=1}^{\infty} \frac{q^{n}}{1+q^{2 n}}, \quad q=e^{\pi i \tau}
$$

Therefore,

$$
\begin{aligned}
\vartheta_{3}^{2}(0 \mid \tau)-\vartheta_{3}^{2}(0 \mid 5 \tau) & =4 \sum_{n=1}^{\infty}\left(\frac{q^{n}}{1+q^{2 n}}-\frac{q^{5 n}}{1+q^{10 n}}\right) \\
& =4 \sum_{n=1}^{\infty}\left(\frac{q^{n}\left(1-q^{2 n}+q^{4 n}-q^{6 n}+q^{8 n}\right)}{1+q^{10 n}}-\frac{q^{5 n}}{1+q^{10 n}}\right) \\
& =4 \sum_{n=0}^{\infty} \frac{q^{n}-q^{3 n}-q^{7 n}+q^{9 n}}{1+q^{10 n}} .
\end{aligned}
$$

This establishes the first part of the identity. To prove the remaining part of the identity, we again appeal to (1.10) (If we look at the last part of the identity (3.1), it would seem that (1.5) is the one to use. However, direct application of (1.5) would result in a good deal of complication).

We need to recall that $[5$, p. 464$]$

$$
\vartheta_{1}(z)=i q^{\frac{1}{4}} e^{-i z} \vartheta_{4}\left(z-\frac{\pi \tau}{2}\right) \quad \text { and } \quad \vartheta_{2}(z)=q^{\frac{1}{4}} e^{-i z} \vartheta_{3}\left(z-\frac{\pi \tau}{2}\right) .
$$

Thus

$$
\frac{\vartheta_{2}(z \mid \tau)}{\vartheta_{1}(z \mid \tau)}=-i \frac{\vartheta_{3}\left(z-\frac{\pi \tau}{2} \mid \tau\right)}{\vartheta_{4}\left(z-\frac{\pi \tau}{2} \mid \tau\right)}
$$


So, from the second identity of (1.11), we have

$$
\begin{aligned}
\frac{i}{2} \vartheta_{3}(0 \mid 5 \tau) \vartheta_{4}(0 \mid 5 \tau) & \left(\frac{\vartheta_{2}\left(\frac{\pi \tau}{2} \mid 5 \tau\right)}{\vartheta_{1}\left(\frac{\pi \tau}{2} \mid 5 \tau\right)}-\frac{\vartheta_{2}\left(\frac{3 \pi \tau}{2} \mid 5 \tau\right)}{\vartheta_{1}\left(\frac{3 \pi \tau}{2} \mid 5 \tau\right)}\right) \\
= & \frac{1}{2} \vartheta_{3}(0 \mid 5 \tau) \vartheta_{4}(0 \mid 5 \tau)\left(\frac{\vartheta_{3}(2 \pi \tau \mid 5 \tau)}{\vartheta_{4}(2 \pi \tau \mid 5 \tau)}-\frac{\vartheta_{3}(\pi \tau \mid 5 \tau)}{\vartheta_{4}(\pi \tau \mid 5 \tau)}\right) \\
= & \sum_{n=1}^{\infty} \frac{q^{n}-q^{3 n}-q^{7 n}+q^{9 n}}{1+q^{10 n}} \\
= & -\frac{1}{2} \frac{\vartheta_{1}^{\prime}(0 \mid 5 \tau) \vartheta_{1}(3 \pi \tau \mid 5 \tau) \vartheta_{1}\left(\frac{\pi \tau}{2} \mid 5 \tau\right) \vartheta_{2}\left(\frac{\pi \tau}{2} \mid 5 \tau\right)}{\vartheta_{4}(2 \pi \tau \mid 5 \tau) \vartheta_{4}(\pi \tau \mid 5 \tau) \vartheta_{3}\left(\frac{3 \pi \tau}{2} \mid 5 \tau\right) \vartheta_{4}\left(\frac{3 \pi \tau}{2} \mid 5 \tau\right)} .
\end{aligned}
$$

Using the triple product identities [5, p. 469] for the theta functions, we can easily see the last quantity in (2.2) becomes

$$
\begin{aligned}
q \prod_{n=1}^{\infty} \frac{\left(1-q^{10 n}\right)^{2}\left(1-q^{10 n-1}\right)\left(1-q^{10 n-4}\right)}{\left(1-q^{10 n-1}\right)\left(1-q^{10 n-3}\right)\left(1-q^{10 n-7}\right)\left(1-q^{10 n-9}\right)} \\
\cdot \frac{\left(1-q^{10 n-6}\right)\left(1-q^{10 n-9}\right)\left(1+q^{10 n-1}\right)\left(1+q^{10 n-9}\right)}{\left(1+q^{10 n-2}\right)\left(1+q^{10 n-8}\right)\left(1-q^{10 n-2}\right)\left(1-q^{10 n-8}\right)} .
\end{aligned}
$$

We now observe that the last two factors in the denominator of the above quantity can be rewritten as

$$
\begin{aligned}
\prod_{n=1}^{\infty}\left(1-q^{10 n-2}\right)\left(1-q^{10 n-8}\right)=\prod_{n=1}^{\infty}\left(1-q^{5 n-1}\right)\left(1-q^{5 n-4}\right)\left(1+q^{5 n-1}\right)\left(1+q^{5 n-4}\right) \\
=\prod_{n=1}^{\infty}\left(1-q^{10 n-1}\right)\left(1-q^{10 n-4}\right)\left(1-q^{10 n-6}\right)\left(1-q^{10 n-9}\right) \\
\cdot\left(1+q^{10 n-1}\right)\left(1+q^{10 n-9}\right)\left(1+q^{10 n-4}\right)\left(1+q^{10 n-6}\right) .
\end{aligned}
$$

Therefore, (2.3) becomes

$$
\begin{aligned}
q \prod_{n=1}^{\infty} & \frac{\left(1-q^{10 n}\right)^{2}}{\left(1-q^{10 n-1}\right)\left(1-q^{10 n-3}\right)\left(1-q^{10 n-7}\right)\left(1-q^{10 n-9}\right)} \\
& \cdot \frac{1}{\left(1+q^{10 n-2}\right)\left(1+q^{10 n-4}\right)\left(1+q^{10 n-6}\right)\left(1+q^{10 n-8}\right)} \\
& =q \prod_{n=1}^{\infty} \frac{\left(1-q^{10 n}\right)^{2}\left(1-q^{10 n-5}\right)\left(1+q^{10 n}\right)}{\left(1-q^{2 n-1}\right)\left(1+q^{2 n}\right)} \\
& =q \prod_{n=1}^{\infty}\left(1+q^{2 n-1}\right)\left(1+q^{5 n}\right)\left(1-q^{5 n}\right)^{2}\left(1+q^{10 n}\right) .
\end{aligned}
$$

This completes the proof of (3.1).

To establish (3.6), we need

$$
d s u=\frac{\pi}{2 K} \csc x-\frac{2 \pi}{K} \sum_{n=0}^{\infty} \frac{q^{2 n+1} \sin (2 n+1) x}{1+q^{2 n+1}} .
$$


This is equivalent to

$$
\vartheta_{2} \vartheta_{4} \frac{\vartheta_{3}(x)}{\vartheta_{1}(x)}=\csc x-4 \sum_{n=0}^{\infty} \frac{q^{2 n+1} \sin (2 n+1) x}{1+q^{2 n+1}} .
$$

From (1.3c), we have

$$
\begin{aligned}
\vartheta_{2} \vartheta_{4} & \left(\frac{\vartheta_{3}(x)}{\vartheta_{1}(x)}+\frac{\vartheta_{3}(y)}{\vartheta_{1}(y)}\right) \\
= & \csc x+\csc y-4 \sum_{n=0}^{\infty} \frac{q^{2 n+1}(\sin (2 n+1) x+\sin (2 n+1) y)}{1+q^{2 n+1}} \\
= & \frac{\vartheta_{1}^{\prime} \vartheta_{1}(x-y) \vartheta_{1}\left(\frac{x+y}{2}\right) \vartheta_{3}\left(\frac{x+y}{2}\right)}{\vartheta_{1}(x) \vartheta_{1}(y) \vartheta_{1}\left(\frac{x-y}{2}\right) \vartheta_{3}\left(\frac{x-y}{2}\right)}
\end{aligned}
$$

We now choose $x=\pi / 10$ and $y=3 \pi / 10$ in (2.4). Using the facts that $\sin \pi / 10=\sqrt{5} / 4-1 / 4$ and $\sin 3 \pi / 10=\sqrt{5} / 4+1 / 4$ we have

$$
\begin{gathered}
2 \sqrt{5}\left(1-\sum_{n=0}^{\infty}(-1)^{n}\left\{\frac{q^{10 n+1}}{1+q^{10 n+1}}+\frac{q^{10 n+3}}{1+q^{10 n+3}}+\frac{q^{10 n+7}}{1+q^{10 n+7}}+\frac{q^{10 n+9}}{1+q^{10 n+9}}\right\}\right) \\
=\csc \frac{\pi}{10}+\csc \frac{3 \pi}{10}-4 \sum_{n=0}^{\infty} \frac{q^{2 n+1}\left(\sin (2 n+1) \frac{\pi}{10}+\sin (2 n+1) \frac{3 \pi}{10}\right)}{1+q^{2 n+1}} \\
=\frac{\vartheta_{1}^{\prime} \vartheta_{1}^{2}\left(\frac{\pi}{5}\right) \vartheta_{3}\left(\frac{\pi}{5}\right)}{\vartheta_{1}^{2}\left(\frac{\pi}{10}\right) \vartheta_{1}\left(\frac{3 \pi}{10}\right) \vartheta_{3}\left(\frac{\pi}{10}\right)} .
\end{gathered}
$$

From the triple product identities, (2.5) becomes

(2.6)

$$
2 \sqrt{5} \prod_{n=1}^{\infty} \frac{\left(1+q^{2 n}\right)\left(1-q^{2 n}\right)^{2}\left(1-q^{2 n} e^{\frac{2 \pi i}{5}}\right)^{2}\left(1-q^{2 n} e^{-\frac{2 \pi i}{5}}\right)^{2}\left(1+q^{2 n-1} e^{\frac{2 \pi i}{5}}\right)\left(1+q^{2 n-1} e^{-\frac{2 \pi i}{5}}\right)}{\left(1+q^{10 n}\right)\left(1-q^{2 n} e^{\frac{\pi i}{5}}\right)\left(1-q^{2 n} e^{-\frac{\pi j}{5}}\right)\left(1+q^{2 n-1} e^{\frac{\pi j}{5}}\right)\left(1+q^{2 n-1} e^{-\frac{\pi i}{5}}\right)}
$$

We note that

$$
\begin{aligned}
& \prod_{n=1}^{\infty}\left(1-q^{2 n} e^{\frac{2 \pi i}{5}}\right)\left(1-q^{2 n} e^{-\frac{2 \pi i}{5}}\right) \\
&=\prod_{1}^{\infty}\left(1-q^{n} e^{\frac{\pi i}{5}}\right)\left(1+q^{n} e^{\frac{\pi i}{5}}\right)\left(1-q^{n} e^{-\frac{\pi i}{5}}\right)\left(1+q^{n} e^{\frac{\pi i}{5}}\right) \\
&=\prod_{n=1}^{\infty}\left(1-q^{2 n} e^{\frac{\pi i}{5}}\right)\left(1-q^{2 n-1} e^{\frac{\pi i}{5}}\right)\left(1+q^{2 n} e^{\frac{\pi i}{5}}\right)\left(1+q^{2 n-1} e^{\frac{\pi i}{5}}\right) \\
& \cdot\left(1-q^{2 n} e^{-\frac{\pi i}{5}}\right)\left(1-q^{2 n-1} e^{-\frac{\pi i}{5}}\right)\left(1+q^{2 n} e^{\frac{\pi i}{5}}\right)\left(1+q^{2 n-1} e^{\frac{\pi i}{5}}\right) .
\end{aligned}
$$

Using (2.7) and the following properties of the fifth roots of unity:

$$
\begin{gathered}
(1+x)\left(1-x e^{\frac{\pi i}{5}}\right)\left(1-x e^{\frac{3 \pi i}{5}}\right)\left(1-x e^{-\frac{\pi i}{5}}\right)\left(1-x e^{-\frac{3 \pi i}{5}}\right)=1+x^{5}, \\
(1-x)\left(1-x e^{\frac{2 \pi i}{5}}\right)\left(1-x e^{\frac{4 \pi i}{5}}\right)\left(1-x e^{-\frac{2 \pi i}{5}}\right)\left(1-x e^{-\frac{4 \pi i}{5}}\right)=1-x^{5},
\end{gathered}
$$


we see that $(2.6)$ becomes

$$
\begin{aligned}
& 2 \sqrt{5} \prod_{n=1}^{\infty} \frac{\left(1-q^{2 n}\right)\left(1+q^{2 n}\right)\left(1+q^{10 n-5}\right)\left(1-q^{10 n}\right)}{\left(1+q^{10 n}\right)\left(1+q^{2 n-1}\right)} \\
& \quad=2 \sqrt{5} \prod_{n=1}^{\infty}\left(1-q^{n}\right)\left(1+q^{2 n}\right)^{2}\left(1-q^{5 n}\right)\left(1+q^{10 n-5}\right)^{2}
\end{aligned}
$$

This completes the proof of (3.6).

\section{A COLLECTION OF 44 LAMBERT SERIES IDENTITIES}

The title of this section is slightly misleading, because the actual number of distinct identities is far fewer. For instance, the identities (3.1) and (3.25) both have the same infinite product, so they are different representations of the same entity. So are the pairs (3.2) and (3.10), (3.3) and (3.16), etc. Furthermore, if one recognizes the fact that the identity (3.13) can be obtained from (3.1) with the replacement of $q$ by $-q$, then, after careful examination, there are only 21 genuinely distinct identities. There are 15 identities associated with the modular forms of weight 1. These are (3.1), (3.2), (3.3), (3.4), (3.5), (3.7), (3.8), (3.22), (3.23), (3.27), (3.28), (3.37), (3.38), (3.39), and (3.40). And six identities representing modular forms of weight 2: (3.11), (3.12), (3.20), (3.31), (3.32), and (3.43).

Despite the repetitions, there are, however, several reasons for us to list these 44 identities. First, the last line of each identity gives a clear indication as to how it is derived. Also, it shows that we have systematically exhausted all the possibilities in obtaining identities arising from this particular approach. Moreover, since many identities can be derived in two different ways, it yields several different representations for that identity. For example, the identities (3.5) and (3.42) show that $5 \vartheta_{4}^{2}(0 \mid 5 \tau)-\vartheta_{4}^{2}(0 \mid \tau)$ can be written in five seemingly different ways. It is not apparent that they all represent the same expression until we recognize that they both have the same infinite product representation. Finally, this table also allows us to see the relations between various identities. For example, if we apply the imaginary transformation to (3.1), it gives (3.9), and replacing the $q$ in (3.9) by $-q$ yields (3.5). Now the application of the imaginary transformation to (3.5) leads to (3.3). So one sees clearly that (3.1), (3.3), (3.5), (3.9), (3.13) and (3.23) are linked by a series of successive applications of the imaginary transformation and the replacement of $q$ by $-q$.

One more clarification needs to be made before we present the table. That is, if one glances at the list, one immediately realizes that these identities are obtained from two types of substitutions: (1) evaluations at $\pi / 10$ and $3 \pi / 10$; (2) replacement of $q$ by $q^{5}$ (or $q^{\frac{5}{2}}$ ) and evaluations at $\pi \tau / 2$ and $3 \pi \tau / 2$ (or $\pi \tau / 4$ and $3 \pi \tau / 4)$. Naturally, one might ask: Are there any other substitutions? Simple experiment shows that there are, indeed, others. But using the basic properties of the theta functions, they invariably are reduced to the abovementioned two types of substitutions and we have already encountered one such instance earlier in (2.2). 
ON THE ADDITIVE FORMULAE OF THE THETA FUNCTIONS

333

$$
\begin{aligned}
& \sum_{n=1}^{\infty} \frac{q^{n}-q^{3 n}-q^{7 n}+q^{9 n}}{1+q^{10 n}}=\frac{1}{4}\left(\vartheta_{3}^{2}(0 \mid \tau)-\vartheta_{3}^{2}(0 \mid 5 \tau)\right) \\
& \quad=q \prod_{n=1}^{\infty}\left(1+q^{2 n-1}\right)\left(1+q^{5 n}\right)\left(1-q^{5 n}\right)^{2}\left(1+q^{10 n}\right) \\
& \quad=\frac{i}{2} \vartheta_{3}(0 \mid 5 \tau) \vartheta_{4}(0 \mid 5 \tau)\left(\frac{\vartheta_{2}}{\vartheta_{1}}\left(\frac{\pi \tau}{2} \mid 5 \tau\right)-\frac{\vartheta_{2}}{\vartheta_{1}}\left(\frac{3 \pi \tau}{2} \mid 5 \tau\right)\right),
\end{aligned}
$$

$$
\begin{aligned}
1+\sum_{n=1}^{\infty} & \frac{q^{n}+q^{3 n}+q^{7 n}+q^{9 n}}{1+q^{10 n}}=\frac{1}{4}\left(\vartheta_{2}^{2}(0 \mid \tau)-\vartheta_{2}^{2}(0 \mid 5 \tau)\right) \frac{\vartheta_{2}\left(0 \mid \frac{\tau}{2}\right)}{\vartheta_{2}\left(0 \mid \frac{5 \tau}{2}\right)} \\
& =\prod_{n=1}^{\infty}\left(1-q^{n}\right)\left(1+q^{n}\right)^{2}\left(1+q^{2 n}\right)\left(1-q^{5 n}\right)\left(1-q^{10 n-5}\right)\left(1+q^{10 n-5}\right) \\
& =\frac{i}{2} \vartheta_{3}(0 \mid 5 \tau) \vartheta_{4}(0 \mid 5 \tau)\left(\frac{\vartheta_{2}}{\vartheta_{1}}\left(\frac{\pi \tau}{2} \mid 5 \tau\right)+\frac{\vartheta_{2}}{\vartheta_{1}}\left(\frac{3 \pi \tau}{2} \mid 5 \tau\right)\right)
\end{aligned}
$$

$$
\begin{aligned}
\sum_{n=0}^{\infty}( & -1)^{n}\left\{\frac{q^{5 n+\frac{1}{2}}}{1-q^{10 n+1}}-\frac{q^{5 n+\frac{3}{2}}}{1-q^{10 n+3}}-\frac{q^{5 n+\frac{7}{2}}}{1-q^{10 n+7}}+\frac{q^{5 n+\frac{9}{2}}}{1-q^{10 n+9}}\right\} \\
= & q^{\frac{1}{2}} \prod_{n=1}^{\infty}\left(1+q^{2 n}\right)\left(1+q^{5 n}\right)\left(1-q^{5 n}\right)^{2}\left(1+q^{10 n-5}\right) \\
& =\frac{1}{4}\left(\vartheta_{2}^{2}(0 \mid \tau)-\vartheta_{2}^{2}(0 \mid 5 \tau)\right) \\
& =\frac{i}{2} \vartheta_{2}(0 \mid 5 \tau) \vartheta_{4}(0 \mid 5 \tau)\left(\frac{\vartheta_{3}}{\vartheta_{1}}\left(\frac{\pi \tau}{2} \mid 5 \tau\right)-\frac{\vartheta_{3}}{\vartheta_{1}}\left(\frac{3 \pi \tau}{2} \mid 5 \tau\right)\right)
\end{aligned}
$$

$$
\begin{aligned}
& \sum_{n=0}^{\infty} \frac{q^{n+\frac{1}{2}}+q^{3 n+\frac{3}{2}}+q^{7 n+\frac{7}{2}}+q^{9 n+\frac{9}{2}}}{1+q^{10 n+5}} \\
& \quad=\frac{1}{4}\left(\vartheta_{3}^{2}(0 \mid \tau)-\vartheta_{3}^{2}(0 \mid 5 \tau)\right) \frac{\vartheta_{2}\left(0 \mid \frac{\tau}{2}\right)}{\vartheta_{2}\left(0 \mid \frac{5 \tau}{2}\right)} \\
& \quad=q^{\frac{1}{2}} \prod_{n=1}^{\infty}\left(1-q^{n}\right)\left(1+q^{n}\right)^{2}\left(1+q^{2 n-1}\right)\left(1-q^{5 n}\right)\left(1-q^{10 n-5}\right)\left(1+q^{10 n}\right) \\
& \quad=\frac{i}{2} \vartheta_{2}(0 \mid 5 \tau) \vartheta_{4}(0 \mid 5 \tau)\left(\frac{\vartheta_{3}}{\vartheta_{1}}\left(\frac{\pi \tau}{2} \mid 5 \tau\right)+\frac{\vartheta_{3}}{\vartheta_{1}}\left(\frac{3 \pi \tau}{2} \mid 5 \tau\right)\right),
\end{aligned}
$$




$$
\begin{aligned}
1+\sum_{n=0}^{\infty}(-1)^{n}\left\{\frac{q^{10 n+1}}{1+q^{10 n+1}}-\frac{q^{10 n+3}}{1+q^{10 n+3}}\right. & \left.-\frac{4 q^{10 n+5}}{1+q^{10 n+5}}-\frac{q^{10 n+7}}{1+q^{10 n+7}}+\frac{q^{10 n+9}}{1+q^{10 n+9}}\right\} \\
= & \prod_{n=1}^{\infty}\left(1-q^{n}\right)^{2}\left(1+q^{n}\right)^{3}\left(1-q^{10 n-5}\right)=\frac{1}{4}\left(5 \vartheta_{4}^{2}(0 \mid 5 \tau)-\vartheta_{4}^{2}(0 \mid \tau)\right) \\
= & \frac{1}{2} \vartheta_{2}(0 \mid \tau) \vartheta_{4}(0 \mid \tau)\left(\frac{\vartheta_{3}}{\vartheta_{1}}\left(\frac{\pi}{10} \mid \tau\right)-\frac{\vartheta_{3}}{\vartheta_{1}}\left(\frac{3 \pi}{10} \mid \tau\right)\right),
\end{aligned}
$$

$$
\begin{aligned}
1- & \sum_{n=0}^{\infty}(-1)^{n}\left\{\frac{q^{10 n+1}}{1+q^{10 n+1}}+\frac{q^{10 n+3}}{1+q^{10 n+3}}+\frac{q^{10 n+7}}{1+q^{10 n+7}}+\frac{q^{10 n+9}}{1+q^{10 n+9}}\right\} \\
& =\prod_{n=1}^{\infty}\left(1-q^{n}\right)\left(1+q^{2 n}\right)^{2}\left(1-q^{5 n}\right)\left(1+q^{10 n-5}\right)^{2} \\
& =\frac{1}{2 \sqrt{5}} \vartheta_{2}(0 \mid \tau) \vartheta_{4}(0 \mid \tau)\left(\frac{\vartheta_{3}}{\vartheta_{1}}\left(\frac{\pi}{10} \mid \tau\right)+\frac{\vartheta_{3}}{\vartheta_{1}}\left(\frac{3 \pi}{10} \mid \tau\right)\right)
\end{aligned}
$$

$$
\begin{aligned}
\sum_{n=0}^{\infty} & \frac{q^{5 n+\frac{1}{2}}}{1-q^{10 n+1}}-\frac{q^{5 n+\frac{3}{2}}}{1-q^{10 n+3}}+\frac{q^{5 n+\frac{7}{2}}}{1-q^{10 n+7}}-\frac{q^{5 n+\frac{9}{2}}}{1-q^{10 n+9}} \\
= & q^{\frac{1}{2}} \prod_{n=1}^{\infty}\left(1+q^{n}\right)\left(1-q^{5 n}\right)^{2}\left(1+q^{5 n}\right)\left(1-q^{5 n-1}\right)\left(1+q^{5 n-2}\right) \\
& \cdot\left(1+q^{5 n-3}\right)\left(1-q^{5 n-4}\right) \\
= & \frac{i}{2} \vartheta_{2}(0 \mid 5 \tau) \vartheta_{3}(0 \mid 5 \tau)\left(\frac{\vartheta_{4}}{\vartheta_{1}}\left(\frac{\pi \tau}{2} \mid 5 \tau\right)-\frac{\vartheta_{4}}{\vartheta_{1}}\left(\frac{3 \pi \tau}{2} \mid 5 \tau\right)\right)
\end{aligned}
$$

$$
\begin{aligned}
\sum_{n=0}^{\infty} \frac{q^{5 n+\frac{1}{2}}}{1-q^{10 n+1}}+\frac{q^{5 n+\frac{3}{2}}}{1-q^{10 n+3}}-\frac{q^{5 n+\frac{7}{2}}}{1-q^{10 n+7}}-\frac{q^{5 n+\frac{\vartheta}{2}}}{1-q^{10 n+9}} \\
=q^{\frac{1}{2}} \prod_{n=1}^{\infty}\left(1+q^{n}\right)\left(1-q^{5 n}\right)^{2}\left(1+q^{5 n}\right)\left(1+q^{5 n-1}\right) \\
\cdot\left(1-q^{5 n-2}\right)\left(1-q^{5 n-3}\right)\left(1+q^{5 n-4}\right) \\
=\frac{i}{2} \vartheta_{2}(0 \mid 5 \tau) \vartheta_{3}(0 \mid 5 \tau)\left(\frac{\vartheta_{4}}{\vartheta_{1}}\left(\frac{\pi \tau}{2} \mid 5 \tau\right)+\frac{\vartheta_{4}}{\vartheta_{1}}\left(\frac{3 \pi \tau}{2} \mid 5 \tau\right)\right),
\end{aligned}
$$




$$
\begin{aligned}
& 1-\sum_{n=0}^{\infty}(-1)^{n}\left\{\frac{q^{10 n+1}}{1-q^{10 n+1}}-\frac{q^{10 n+3}}{1-q^{10 n+3}}-\frac{4 q^{10 n+5}}{1-q^{10 n+5}}\right. \\
& \left.-\frac{q^{10 n+7}}{1-q^{10 n+7}}+\frac{q^{10 n+9}}{1-q^{10 n+9}}\right\} \\
& =\prod_{n=1}^{\infty}\left(1+q^{n}\right)\left(1-q^{n}\right)^{2}\left(1+q^{2 n}\right)\left(1+q^{10 n-5}\right) \\
& =\frac{1}{4}\left(5 \vartheta_{3}^{2}(0 \mid 5 \tau)-\vartheta_{3}^{2}(0 \mid \tau)\right) \\
& =\frac{1}{2} \vartheta_{2}(0 \mid \tau) \vartheta_{3}(0 \mid \tau)\left(\frac{\vartheta_{4}}{\vartheta_{1}}\left(\frac{\pi}{10} \mid \tau\right)-\frac{\vartheta_{4}}{\vartheta_{1}}\left(\frac{3 \pi}{10} \mid \tau\right)\right) \text {, } \\
& 1+\sum_{n=0}^{\infty}(-1)^{n}\left\{\frac{q^{10 n+1}}{1-q^{10 n+1}}+\frac{q^{10 n+3}}{1-q^{10 n+3}}+\frac{q^{10 n+7}}{1-q^{10 n+7}}+\frac{q^{10 n+9}}{1-q^{10 n+9}}\right\} \\
& =\prod_{n=1}^{\infty}\left(1-q^{n}\right)\left(1+q^{n}\right)^{2}\left(1+q^{2 n}\right)\left(1-q^{5 n}\right)\left(1-q^{10 n-5}\right)\left(1+q^{10 n-5}\right) \\
& =\frac{1}{2 \sqrt{5}} \vartheta_{2}(0 \mid \tau) \vartheta_{3}(0 \mid \tau)\left(\frac{\vartheta_{4}}{\vartheta_{1}}\left(\frac{\pi}{10} \mid \tau\right)+\frac{\vartheta_{4}}{\vartheta_{1}}\left(\frac{3 \pi}{10} \mid \tau\right)\right) \text {, } \\
& \sum_{n=0}^{\infty} \frac{n\left(q^{n}-q^{3 n}-q^{7 n}+q^{9 n}\right)}{1-q^{10 n}} \\
& =q \prod_{n=1}^{\infty}\left(1-q^{n}\right)\left(1+q^{n}\right)^{3}\left(1+q^{5 n}\right)\left(1-q^{5 n}\right)^{3} \\
& =\frac{1}{16}\left(\vartheta_{2}^{3}\left(0 \mid \frac{\tau}{2}\right) \vartheta_{2}\left(0 \mid \frac{5 \tau}{2}\right)-\vartheta_{2}\left(0 \mid \frac{\tau}{2}\right) \vartheta_{2}^{3}\left(0 \mid \frac{5 \tau}{2}\right)\right) \\
& =\frac{1}{4}\left\{\left(\frac{\vartheta_{1}^{\prime}}{\vartheta_{1}}\right)^{\prime}\left(\frac{\pi \tau}{2} \mid 5 \tau\right)-\left(\frac{\vartheta_{1}^{\prime}}{\vartheta_{1}}\right)^{\prime}\left(\frac{3 \pi \tau}{2} 5 \tau\right)\right\} \text {, } \\
& 1-\sum_{n=0}^{\infty}(-1)^{n}\left\{\frac{(5 n+1) q^{5 n+1}}{1-q^{5 n+1}}+\frac{(5 n+2) q^{5 n+2}}{1-q^{5 n+2}}\right. \\
& \left.-\frac{(5 n+3) q^{5 n+3}}{1-q^{5 n+3}}-\frac{(5 n+4) q^{5 n+4}}{1-q^{5 n+4}}\right\} \\
& =\prod_{n=1}^{\infty}\left(1-q^{n}\right)\left(1-q^{2 n}\right)^{2}\left(1-q^{5 n}\right)\left(1-q^{10 n-5}\right)^{2} \\
& =\frac{1}{4 \sqrt{5}}\left\{\left(\frac{\vartheta_{1}^{\prime}}{\vartheta_{1}}\right)^{\prime}\left(\frac{3 \pi}{10} \mid \frac{\tau}{2}\right)-\left(\frac{\vartheta_{1}^{\prime}}{\vartheta_{1}}\right)^{\prime}\left(\frac{\pi}{10} \mid \frac{\tau}{2}\right)\right\} \\
& =\frac{1}{4}\left(5 \vartheta_{4}(0 \mid \tau) \vartheta_{4}^{3}(0 \mid 5 \tau)-\vartheta_{4}^{3}(0 \mid \tau) \vartheta_{4}(0 \mid 5 \tau)\right) \text {, }
\end{aligned}
$$


(3.13)

$$
\begin{aligned}
\sum_{n=0}^{\infty}( & -1)^{n}\left\{\frac{q^{10 n+1}}{1+q^{10 n+1}}-\frac{q^{10 n+3}}{1+q^{10 n+3}}-\frac{q^{10 n+7}}{1+q^{10 n+7}}+\frac{q^{10 n+9}}{1+q^{10 n+9}}\right\} \\
& =\sum_{n=1}^{\infty}(-1)^{n-1}\left\{\frac{q^{n}-q^{3 n}-q^{7 n}+q^{9 n}}{1+q^{10 n}}\right\} \\
& =q \prod_{n=1}^{\infty}\left(1-q^{2 n-1}\right)\left(1-q^{5 n}\right)^{2}\left(1+q^{5 n}\right)^{3} \\
& =\frac{1}{4}\left(\vartheta_{4}^{2}(0 \mid 5 \tau)-\vartheta_{4}^{2}(0 \mid \tau)\right) \\
& =\frac{i}{2} \vartheta_{3}(0 \mid 5 \tau) \vartheta_{4}(0 \mid 5 \tau)\left(\frac{\vartheta_{1}}{\vartheta_{2}}\left(\frac{\pi \tau}{2} \mid 5 \tau\right)-\frac{\vartheta_{1}}{\vartheta_{2}}\left(\frac{3 \pi \tau}{2} \mid 5 \tau\right)\right) \\
1- & \sum_{n=0}^{\infty}(-1)^{n}\left\{\frac{q^{10 n+1}}{1+q^{10 n+1}}+\frac{q^{10 n+3}}{q^{10 n+3}}+\frac{q^{10 n+7}}{q^{10 n+7}}+\frac{q^{10 n+9}}{1+q^{10 n+9}}\right\} \\
& =1+\sum_{n=1}^{\infty}(-1)^{n}\left\{\frac{q^{n}+q^{3 n}+q^{7 n}+q^{9 n}}{1+q^{10 n}}\right\} \\
& =\prod_{n=1}^{\infty}\left(1-q^{n}\right)\left(1+q^{2 n}\right)^{2}\left(1-q^{5 n}\right)\left(1+q^{10 n-5}\right)^{2} \\
& =\frac{1}{2 i} \vartheta_{3}(0 \mid 5 \tau) \vartheta_{4}(0 \mid 5 \tau)\left(\frac{\vartheta_{1}}{\vartheta_{2}}\left(\frac{\pi \tau}{2} \mid 5 \tau\right)+\frac{\vartheta_{1}}{\vartheta_{2}}\left(\frac{3 \pi \tau}{2} \mid 5 \tau\right)\right) \\
&
\end{aligned}
$$

(3.15)

$$
\begin{aligned}
& \sum_{n=0}^{\infty} \frac{(-1)^{n}\left(q^{n+\frac{1}{2}}-q^{3 n+\frac{3}{2}}-q^{7 n+\frac{7}{2}}+q^{9 n+\frac{9}{2}}\right)}{1-q^{10 n+5}} \\
& \quad=q^{\frac{1}{2}} \prod_{n=1}^{\infty}\left(1-q^{n}\right)\left(1-q^{2 n-1}\right)\left(1+q^{2 n}\right)\left(1+q^{5 n}\right)\left(1+q^{10 n-5}\right)\left(1-q^{10 n}\right) \\
& \quad=\frac{1}{2} \vartheta_{2}(0 \mid 5 \tau) \vartheta_{3}(0 \mid 5 \tau)\left(\frac{\vartheta_{3}}{\vartheta_{2}}\left(\frac{\pi \tau}{2} \mid 5 \tau\right)-\frac{\vartheta_{3}}{\vartheta_{2}}\left(\frac{3 \pi \tau}{2} \mid 5 \tau\right)\right)
\end{aligned}
$$

$$
\begin{gathered}
\sum_{n=0}^{\infty} \frac{q^{5 n+\frac{1}{2}}}{1+q^{10 n+1}}+\frac{q^{5 n+\frac{3}{2}}}{1+q^{10 n+3}}+\frac{q^{5 n+\frac{7}{2}}}{1+q^{10 n+7}}+\frac{q^{5 n+\frac{9}{2}}}{1+q^{10 n+9}} \\
=q^{\frac{1}{2}} \prod_{n=1}^{\infty}\left(1+q^{2 n}\right)\left(1-q^{5 n}\right)^{2}\left(1+q^{5 n}\right)\left(1+q^{10 n-5}\right) \\
\quad=\frac{1}{2} \vartheta_{2}(0 \mid 5 \tau) \vartheta_{3}(0 \mid 5 \tau)\left(\frac{\vartheta_{3}}{\vartheta_{2}}\left(\frac{\pi \tau}{2} \mid 5 \tau\right)+\frac{\vartheta_{3}}{\vartheta_{2}}\left(\frac{3 \pi \tau}{2} \mid 5 \tau\right)\right)
\end{gathered}
$$




$$
\begin{aligned}
\sum_{n=0}^{\infty}(-1)^{n} & \left\{\frac{q^{5 n+\frac{1}{2}}}{1+q^{10 n+1}}-\frac{q^{5 n+\frac{3}{2}}}{1+q^{10 n+3}}+\frac{q^{5 n+\frac{7}{2}}}{1+q^{10 n+7}}-\frac{q^{5 n+\frac{9}{2}}}{1+q^{10 n+9}}\right\} \\
= & q^{\frac{1}{2}} \prod_{n=1}^{\infty}\left(1-q^{2 n-1}\right)\left(1+q^{2 n}\right)\left(1-q^{10 n}\right)^{2}\left(1-q^{10 n-1}\right)\left(1-q^{10 n-2}\right)\left(1+q^{10 n-3}\right) \\
& \cdot\left(1+q^{10 n-4}\right)\left(1+q^{10 n-5}\right)\left(1+q^{10 n-6}\right)\left(1+q^{10 n-7}\right)\left(1-q^{10 n-8}\right)\left(1-q^{10 n-9}\right) \\
= & \frac{1}{2} \vartheta_{2}(0 \mid 5 \tau) \vartheta_{4}(0 \mid 5 \tau)\left(\frac{\vartheta_{4}}{\vartheta_{2}}\left(\frac{\pi \tau}{2} \mid 5 \tau\right)-\frac{\vartheta_{4}}{\vartheta_{2}}\left(\frac{3 \pi \tau}{2} \mid 5 \tau\right)\right),
\end{aligned}
$$

$$
\begin{aligned}
\sum_{n=0}^{\infty}(-1)^{n} & \left\{\frac{q^{5 n+\frac{1}{2}}}{1+q^{10 n+1}}+\frac{q^{5 n+\frac{3}{2}}}{1+q^{10 n+3}}-\frac{q^{5 n+\frac{7}{2}}}{1+q^{10 n+7}}-\frac{q^{5 n+\frac{9}{2}}}{1+q^{10 n+9}}\right\} \\
= & q^{\frac{1}{2}} \prod_{n=1}^{\infty}\left(1-q^{2 n-1}\right)\left(1+q^{2 n}\right)\left(1-q^{10 n}\right)^{2}\left(1+q^{10 n-1}\right)\left(1+q^{10 n-2}\right)\left(1-q^{10 n-3}\right) \\
& \cdot\left(1-q^{10 n-4}\right)\left(1+q^{10 n-5}\right)\left(1-q^{10 n-6}\right)\left(1-q^{10 n-7}\right)\left(1+q^{10 n-8}\right)\left(1+q^{10 n-9}\right) \\
= & \frac{1}{2} \vartheta_{2}(0 \mid 5 \tau) \vartheta_{4}(0 \mid 5 \tau)\left(\frac{\vartheta_{4}}{\vartheta_{2}}\left(\frac{\pi \tau}{2} \mid 5 \tau\right)+\frac{\vartheta_{4}}{\vartheta_{2}}\left(\frac{3 \pi \tau}{2} \mid 5 \tau\right)\right)
\end{aligned}
$$

$$
\begin{aligned}
& \sum_{n=0}^{\infty} \frac{q^{10 n+1}}{\left(1+q^{10 n+1}\right)^{2}}-\frac{q^{10 n+3}}{\left(1+q^{10 n+3}\right)^{2}}-\frac{q^{10 n+7}}{\left(1+q^{10 n+7}\right)^{2}}+\frac{q^{10 n+9}}{\left(1+q^{10 n+9}\right)^{2}} \\
& \quad=\sum_{n=1}^{\infty} \frac{(-1)^{n-1} n\left(q^{n}-q^{3 n}-q^{7 n}+q^{9 n}\right)}{1-q^{10 n}} \\
& \quad=q \prod_{n=1}^{\infty}\left(1-q^{n}\right)\left(1-q^{2 n-1}\right)^{2}\left(1+q^{2 n}\right)^{2}\left(1+q^{10 n-5}\right)^{2}\left(1-q^{10 n}\right)^{3} \\
& \quad=\frac{1}{4}\left\{\left(\frac{\vartheta_{2}^{\prime}}{\vartheta_{2}}\right)^{\prime}\left(\frac{3 \pi \tau}{2} \mid 5 \tau\right)-\left(\frac{\vartheta_{2}^{\prime}}{\vartheta_{2}}\right)^{\prime}\left(\frac{\pi \tau}{2} \mid 5 \tau\right)\right\},
\end{aligned}
$$

$$
\begin{aligned}
1-5 & \sum_{n=1}^{\infty} \frac{(5 n+1) q^{5 n+1}}{1-q^{5 n+1}}-\frac{(5 n+2) q^{5 n+2}}{1-q^{5 n+2}}-\frac{(5 n+3) q^{5 n+3}}{1-q^{5 n+3}}+\frac{(5 n+4) q^{5 n+4}}{1-q^{5 n+4}} \\
& =\prod_{n=1}^{\infty} \frac{\left(1-q^{n}\right)^{5}}{1-q^{5 n}}=\frac{1}{16}\left(\frac{\vartheta_{2}^{5}\left(0 \mid \frac{\tau}{4}\right)}{\vartheta_{2}\left(0 \mid \frac{5 \tau}{4}\right)}-5 \vartheta_{2}^{3}\left(0 \mid \frac{\tau}{4}\right) \vartheta_{2}\left(0 \mid \frac{5 \tau}{4}\right)\right) \\
& =\frac{\sqrt{5}}{4}\left\{\left(\frac{\vartheta_{2}^{\prime}}{\vartheta_{2}}\right)^{\prime}\left(\frac{\pi}{10} \mid \frac{\tau}{2}\right)-\left(\frac{\vartheta_{2}^{\prime}}{\vartheta_{2}}\right)^{\prime}\left(\frac{3 \pi}{10} \mid \frac{\tau}{2}\right)\right\},
\end{aligned}
$$




$$
\begin{gathered}
\sum_{n=0}^{\infty}(-1)^{n} \frac{q^{2 n+1}-q^{4 n+2}+q^{6 n+3}-q^{8 n+4}}{1+q^{10 n+5}}=\frac{1}{4}\left(\vartheta_{4}^{2}(0 \mid 5 \tau)-\vartheta_{4}^{2}(0 \mid \tau)\right) \\
=q \prod_{n=1}^{\infty}\left(1-q^{2 n-1}\right)\left(1-q^{5 n}\right)^{2}\left(1+q^{5 n}\right)^{3} \\
=\frac{i}{2} \vartheta_{2}(0 \mid 5 \tau) \vartheta_{4}(0 \mid 5 \tau)\left(\frac{\vartheta_{1}}{\vartheta_{3}}\left(\frac{\pi \tau}{2} \mid 5 \tau\right)-\frac{\vartheta_{1}}{\vartheta_{3}}\left(\frac{3 \pi \tau}{2} \mid 5 \tau\right)\right), \\
\sum_{n=0}^{\infty}(-1)^{n} \frac{\left(q^{2 n+1}+q^{4 n+2}-q^{6 n+3}-q^{8 n+4}\right)}{1+q^{10 n+5}} \\
=q \prod_{n=1}^{\infty}\left(1-q^{n}\right)\left(1+q^{2 n-1}\right)^{2}\left(1-q^{5 n}\right)\left(1+q^{10 n}\right)^{2} \\
=\frac{1}{2 i} \vartheta_{2}(0 \mid 5 \tau) \vartheta_{4}(0 \mid 5 \tau)\left(\frac{\vartheta_{1}}{\vartheta_{3}}\left(\frac{\pi \tau}{2} \mid 5 \tau\right)+\frac{\vartheta_{1}}{\vartheta_{3}}\left(\frac{3 \pi \tau}{2} \mid 5 \tau\right)\right)
\end{gathered}
$$

$$
\begin{aligned}
& \sum_{n=0}^{\infty} \frac{q^{5 n+\frac{1}{2}}}{1+q^{10 n+1}}+\frac{q^{5 n+\frac{3}{2}}}{1+q^{10 n+3}}-\frac{4 q^{5 n+\frac{5}{2}}}{1+q^{10 n+5}}+\frac{q^{5 n+\frac{7}{2}}}{1+q^{10 n+7}}+\frac{q^{5 n+\frac{\vartheta}{2}}}{1+q^{10 n+9}} \\
& \quad=q^{\frac{1}{2}} \prod_{n=1}^{\infty}\left(1-q^{n}\right)^{2}\left(1+q^{n}\right)\left(1+q^{2 n-1}\right)\left(1+q^{10 n}\right) \\
& \quad=\frac{1}{4}\left(\vartheta_{2}^{2}(0 \mid 5 \tau)-5 \vartheta_{2}^{2}(0 \mid 5 \tau)\right) \\
& \quad=\frac{1}{2} \vartheta_{2}(0 \mid \tau) \vartheta_{4}(0 \mid \tau)\left(\frac{\vartheta_{1}}{\vartheta_{3}}\left(\frac{3 \pi}{10} \mid \tau\right)-\frac{\vartheta_{1}}{\vartheta_{3}}\left(\frac{\pi}{10} \mid \tau\right)\right)
\end{aligned}
$$

(3.24)

$$
\begin{aligned}
& \sum_{n=0}^{\infty} \frac{q^{5 n+\frac{1}{2}}}{1+q^{10 n+1}}-\frac{q^{5 n+\frac{3}{2}}}{1+q^{10 n+3}}-\frac{q^{5 n+\frac{1}{2}}}{1+q^{10 n+7}}+\frac{q^{5 n+\frac{9}{2}}}{1+q^{10 n+9}} \\
& \quad=q^{\frac{1}{2}} \prod_{n=1}^{\infty}\left(1-q^{n}\right)\left(1-q^{2 n-1}\right)\left(1+q^{2 n}\right)\left(1-q^{5 n}\right)\left(1+q^{5 n}\right)^{2}\left(1+q^{10 n-5}\right) \\
& \quad=\frac{1}{2 \sqrt{5}} \vartheta_{2}(0 \mid \tau) \vartheta_{4}(0 \mid \tau)\left(\frac{\vartheta_{1}}{\vartheta_{3}}\left(\frac{3 \pi}{10} \mid \tau\right)+\frac{\vartheta_{1}}{\vartheta_{3}}\left(\frac{\pi}{10} \mid \tau\right)\right),
\end{aligned}
$$

$$
\begin{aligned}
& \sum_{n=0}^{\infty} \frac{q^{5 n+1}}{1+q^{10 n+2}}+\frac{q^{5 n+2}}{1+q^{10 n+4}}+\frac{q^{5 n+3}}{1+q^{10 n+6}}+\frac{q^{5 n+4}}{1+q^{10 n+8}} \\
& \quad=\sum_{n=0}^{\infty}(-1)^{n} \frac{q^{2 n+1}+q^{4 n+2}+q^{6 n+3}+q^{8 n+4}}{1-q^{10 n+5}} \\
& \quad=q \prod_{n=1}^{\infty}\left(1+q^{2 n-1}\right)\left(1+q^{5 n}\right)\left(1-q^{5 n}\right)^{2}\left(1+q^{10 n}\right) \\
& \quad=\frac{1}{2} \vartheta_{2}(0 \mid 5 \tau) \vartheta_{3}(0 \mid 5 \tau)\left(\frac{\vartheta_{2}}{\vartheta_{3}}\left(\frac{\pi \tau}{2} \mid 5 \tau\right)+\frac{\vartheta_{2}}{\vartheta_{3}}\left(\frac{3 \pi \tau}{2} \mid 5 \tau\right)\right),
\end{aligned}
$$




$$
\begin{aligned}
& \sum_{n=0}^{\infty}(-1)^{n} \frac{q^{2 n+1}-q^{4 n+2}-q^{6 n+3}+q^{8 n+4}}{1-q^{10 n+5}} \\
& =q \prod_{n=1}^{\infty}\left(1-q^{n}\right)\left(1-q^{2 n-1}\right)\left(1+q^{2 n-1}\right)\left(1-q^{5 n}\right)\left(1+q^{5 n}\right)^{2}\left(1+q^{10 n}\right) \\
& =\frac{1}{2} \vartheta_{2}(0 \mid 5 \tau) \vartheta_{3}(0 \mid 5 \tau)\left(\frac{\vartheta_{2}}{\vartheta_{3}}\left(\frac{3 \pi \tau}{2} \mid 5 \tau\right)-\frac{\vartheta_{2}}{\vartheta_{3}}\left(\frac{\pi \tau}{2} \mid 5 \tau\right)\right) \\
& \sum_{n=0}^{\infty}(-1)^{n}\left\{\frac{q^{5 n+1}}{1+q^{5 n+1}}-\frac{q^{5 n+2}}{1+q^{5 n+2}}-\frac{q^{5 n+3}}{1+q^{5 n+3}}+\frac{q^{5 n+4}}{1+q^{5 n+4}}\right\} \\
& =\sum_{n=0}^{\infty} \frac{(-1)^{n-1}\left(q^{n}-q^{2 n}-q^{3 n}+q^{4 n}\right)}{1+q^{5 n}} \\
& =q \prod_{n=1}^{\infty}\left(1-q^{2 n-1}\right)\left(1+q^{5 n}\right)\left(1-q^{5 n}\right)^{2}\left(1-q^{10 n-1}\right) \\
& \cdot\left(1-q^{10 n-2}\right)\left(1-q^{10 n-8}\right)\left(1-q^{10 n-9}\right) \\
& =\frac{1}{2} \vartheta_{3}\left(0 \mid \frac{5 \tau}{2}\right) \vartheta_{4}\left(0 \mid \frac{5 \tau}{2}\right)\left(\frac{\vartheta_{4}}{\vartheta_{3}}\left(\frac{\pi \tau}{4} \mid \frac{5 \tau}{2}\right)-\frac{\vartheta_{4}}{\vartheta_{3}}\left(\frac{3 \pi \tau}{4} \mid \frac{5 \tau}{2}\right)\right) \text {, } \\
& 1+\sum_{n=0}^{\infty}(-1)^{n} \frac{\left.q^{n}+q^{2 n}+q^{3 n}+q^{4 n}\right)}{1+q^{5 n}} \\
& =\prod_{n=1}^{\infty}\left(1-q^{2 n-1}\right)\left(1+q^{5 n}\right)\left(1-q^{5 n}\right)^{2}\left(1-q^{10 n-3}\right) \\
& \cdot\left(1-q^{10 n-4}\right)\left(1-q^{10 n-6}\right)\left(1-q^{10 n-7}\right) \\
& =\frac{1}{2} \vartheta_{3}\left(0 \mid \frac{5 \tau}{2}\right) \vartheta_{4}\left(0 \mid \frac{5 \tau}{2}\right)\left(\frac{\vartheta_{4}}{\vartheta_{3}}\left(\frac{\pi \tau}{4} \mid \frac{5 \tau}{2}\right)+\frac{\vartheta_{4}}{\vartheta_{3}}\left(\frac{3 \pi \tau}{4} \mid \frac{5 \tau}{2}\right)\right) \text {, } \\
& \sum_{n=0}^{\infty} \frac{q^{5 n+1}}{1+q^{10 n+2}}-\frac{q^{5 n+2}}{1+q^{10 n+4}}-\frac{q^{5 n+3}}{1+q^{10 n+6}}+\frac{q^{5 n+4}}{1+q^{10 n+8}} \\
& =q \prod_{n=1}^{\infty}\left(1-q^{n}\right)\left(1-q^{2 n-1}\right)\left(1+q^{2 n-1}\right)\left(1-q^{5 n}\right)\left(1+q^{5 n}\right)^{2}\left(1+q^{10 n}\right) \\
& =\frac{1}{4}\left(\vartheta_{3}^{2}(0 \mid \tau)-\vartheta_{3}^{2}(0 \mid 5 \tau)\right) \frac{\vartheta_{4}(0 \mid \tau)}{\vartheta_{4}(0 \mid 5 \tau)} \\
& =\frac{1}{2 \sqrt{5}} \vartheta_{3}(0 \mid \tau) \vartheta_{4}(0 \mid \tau)\left(\frac{\vartheta_{4}}{\vartheta_{3}}\left(\frac{3 \pi}{10} \mid \tau\right)-\frac{\vartheta_{4}}{\vartheta_{3}}\left(\frac{\pi}{10} \mid \tau\right)\right) \text {, } \\
& 1-\sum_{n=0}^{\infty} \frac{q^{5 n+1}}{1+q^{10 n+2}}+\frac{q^{5 n+2}}{1+q^{10 n+4}}+\frac{q^{5 n+3}}{1+q^{10 n+6}}+\frac{q^{5 n+4}}{1+q^{10 n+8}} \\
& =\prod_{n=1}^{\infty}\left(1-q^{n}\right)^{2}\left(1+q^{n}\right)\left(1+q^{2 n}\right)\left(1+q^{10 n-5}\right) \\
& =\frac{1}{2} \vartheta_{3}(0 \mid \tau) \vartheta_{4}(0 \mid \tau)\left(\frac{\vartheta_{4}}{\vartheta_{3}}\left(\frac{\pi}{10} \mid \tau\right)+\frac{\vartheta_{4}}{\vartheta_{3}}\left(\frac{3 \pi}{10} \mid \tau\right)\right),
\end{aligned}
$$




$$
\begin{aligned}
& \sum_{n=0}^{\infty} \frac{q^{5 n+1}}{\left(1+q^{5 n+1}\right)^{2}}-\frac{q^{5 n+2}}{\left(1+q^{5 n+2}\right)^{2}}-\frac{q^{5 n+3}}{\left(1+q^{5 n+3}\right)^{2}}+\frac{q^{5 n+4}}{\left(1+q^{5 n+4}\right)^{2}} \\
& =\sum_{n=1}^{\infty}(-1)^{n-1} \frac{n\left(q^{n}-q^{2 n}-q^{3 n}+q^{4 n}\right)}{1-q^{5 n}} \\
& =q \prod_{n=1}^{\infty}\left(1-q^{n}\right)\left(1-q^{2 n-1}\right)^{2}\left(1-q^{5 n}\right)^{3}\left(1+q^{5 n}\right)^{2} \\
& =\frac{1}{4} \vartheta_{4}(0 \mid \tau) \vartheta_{4}(0 \mid 5 \tau)\left(\vartheta_{4}^{2}(0 \mid \tau)-\vartheta_{4}^{2}(0 \mid 5 \tau)\right) \\
& =\frac{1}{4}\left\{\left(\frac{\vartheta_{3}^{\prime}}{\vartheta_{3}}\right)^{\prime}\left(\frac{\pi \tau}{4} \mid \frac{5 \tau}{2}\right)-\left(\frac{\vartheta_{3}^{\prime}}{\vartheta_{3}}\right)^{\prime}\left(\frac{3 \pi \tau}{4} \mid \frac{5 \tau}{2}\right)\right\} \text {, } \\
& \sum_{n=0}^{\infty} \frac{(5 n+1) q^{5 n+1}}{1-q^{10 n+2}}-\frac{(5 n+2) q^{5 n+2}}{1-q^{10 n+4}}-\frac{(5 n+3) q^{5 n+3}}{1-q^{10 n+6}}+\frac{(5 n+4) q^{5 n+4}}{1-q^{10 n+8}} \\
& =q \prod_{n=1}^{\infty}\left(1-q^{n}\right)^{3}\left(1+q^{n}\right)\left(1-q^{5 n}\right)\left(1+q^{5 n}\right)^{3} \\
& =\frac{1}{4 \sqrt{5}}\left\{\left(\frac{\vartheta_{3}^{\prime}}{\vartheta_{3}}\right)^{\prime}\left(\frac{3 \pi}{10} \mid \tau\right)-\left(\frac{\vartheta_{3}^{\prime}}{\vartheta_{3}}\right)^{\prime}\left(\frac{\pi}{10} \mid \tau\right)\right\}, \\
& \sum_{n=0}^{\infty} \frac{q^{5 n+1}}{1-q^{10 n+2}}-\frac{q^{5 n+2}}{1-q^{10 n+4}}+\frac{q^{5 n+3}}{1-q^{10 n+6}}-\frac{q^{5 n+4}}{1-q^{10 n+8}} \\
& =\sum_{n=0}^{\infty} \frac{q^{2 n+1}-q^{4 n+2}+q^{6 n+3}-q^{8 n+4}}{1-q^{10 n+5}} \\
& =q \prod_{n=1}^{\infty} \frac{\left(1-q^{10 n}\right)^{3}\left(1-q^{5 n-1}\right)\left(1+q^{5 n-2}\right)\left(1+q^{5 n-3}\right)\left(1-q^{5 n-4}\right)}{\left(1-q^{2 n}\right)} \\
& =\frac{1}{2} \vartheta_{2}(0 \mid 5 \tau) \vartheta_{3}(0 \mid 5 \tau)\left(\frac{\vartheta_{1}}{\vartheta_{4}}\left(\frac{3 \pi \tau}{2} \mid 5 \tau\right)-\frac{\vartheta_{1}}{\vartheta_{4}}\left(\frac{\pi \tau}{2} \mid 5 \tau\right)\right) \text {, } \\
& \sum_{n=0}^{\infty} \frac{q^{2 n+1}+q^{4 n+2}-q^{6 n+3}-q^{8 n+4}}{1-q^{10 n+5}} \\
& =q \prod_{n=1}^{\infty} \frac{\left(1-q^{10 n}\right)^{3}\left(1+q^{5 n-1}\right)\left(1-q^{5 n-2}\right)\left(1-q^{5 n-3}\right)\left(1+q^{5 n-4}\right)}{\left(1-q^{2 n}\right)} \\
& =\frac{1}{2} \vartheta_{2}(0 \mid 5 \tau) \vartheta_{3}(0 \mid 5 \tau)\left(\frac{\vartheta_{1}}{\vartheta_{4}}\left(\frac{\pi \tau}{2} \mid 5 \tau\right)+\frac{\vartheta_{1}}{\vartheta_{4}}\left(\frac{3 \pi \tau}{2} \mid 5 \tau\right)\right) \text {, }
\end{aligned}
$$


ON THE ADDITIVE FORMULAE OF THE THETA FUNCTIONS

341

(3.35)

$$
\begin{aligned}
& \sum_{n=0}^{\infty}(-1)^{n}\left\{\frac{q^{5 n+\frac{1}{2}}}{1-q^{10 n+1}}-\frac{q^{5 n+\frac{3}{2}}}{1-q^{10 n+3}}-\frac{4 q^{5 n+\frac{5}{2}}}{1-q^{10 n+5}}-\frac{q^{5 n+\frac{7}{2}}}{1-q^{10 n-7}}+\frac{q^{5 n+\frac{9}{2}}}{1-q^{10 n+9}}\right\} \\
& =q^{\frac{1}{2}} \prod_{n=1}^{\infty}\left(1+q^{n}\right)\left(1-q^{2 n}\right)\left(1+q^{2 n-1}\right)\left(1+q^{10 n}\right) \\
& \quad=\frac{1}{2} \vartheta_{2}(0 \mid \tau) \vartheta_{3}(0 \mid \tau)\left(\frac{\vartheta_{1}}{\vartheta_{4}}\left(\frac{3 \pi}{10} \mid \tau\right)-\frac{\vartheta_{1}}{\vartheta_{4}}\left(\frac{\pi}{10} \mid \tau\right)\right),
\end{aligned}
$$

(3.36)

$$
\begin{aligned}
\sum_{n=0}^{\infty}(-1)^{n} & \left\{\frac{q^{5 n+\frac{1}{2}}}{1-q^{10 n+1}}+\frac{q^{5 n+\frac{3}{2}}}{1-q^{10 n+3}}+\frac{q^{5 n+\frac{7}{2}}}{1-q^{10 n+7}}+\frac{q^{5 n+\frac{9}{2}}}{1-q^{10 n+9}}\right\} \\
= & q^{\frac{1}{2}} \prod_{n=1}^{\infty}\left(1-q^{n}\right)\left(1+q^{n}\right)^{2}\left(1+q^{2 n-1}\right)\left(1-q^{5 n}\right)\left(1-q^{10 n-5}\right)\left(1+q^{10 n}\right) \\
& =\frac{1}{2 \sqrt{5}} \vartheta_{2}(0 \mid \tau) \vartheta_{3}(0 \mid \tau)\left(\frac{\vartheta_{1}}{\vartheta_{4}}\left(\frac{\pi}{10} \mid \tau\right)+\frac{\vartheta_{1}}{\vartheta_{4}}\left(\frac{3 \pi}{10} \mid \tau\right)\right)
\end{aligned}
$$

(3.37)

$$
\begin{aligned}
\sum_{n=0}^{\infty} \frac{q^{2 n+1}-q^{4 n+2}-q^{6 n+3}+q^{8 n+4}}{1+q^{10 n+5}} & \\
= & q \prod_{n=1}^{\infty} \frac{\left(1-q^{10 n}\right)^{3}}{1-q^{2 n}}\left(1-q^{10 n-1}\right)\left(1-q^{10 n-2}\right)\left(1+q^{10 n-3}\right)\left(1+q^{10 n-4}\right) \\
& \cdot\left(1+q^{10 n-6}\right)\left(1+q^{10 n-7}\right)\left(1-q^{10 n-8}\right)\left(1-q^{10 n-9}\right) \\
= & \frac{1}{2} \vartheta_{2}(0 \mid 5 \tau) \vartheta_{4}(0 \mid 5 \tau)\left(\frac{\vartheta_{2}}{\vartheta_{4}}\left(\frac{3 \pi \tau}{2} \mid 5 \tau\right)-\frac{\vartheta_{2}}{\vartheta_{4}}\left(\frac{\pi \tau}{2} \mid 5 \tau\right)\right)
\end{aligned}
$$

(3.38)

$$
\begin{aligned}
\sum_{n=0}^{\infty} & \frac{q^{2 n+1}+q^{4 n+2}+q^{6 n+3}+q^{8 n+4}}{1+q^{10 n+5}} \\
= & q \prod_{n=1}^{\infty} \frac{\left(1-q^{10 n}\right)^{3}}{1-q^{2 n}}\left(1+q^{10 n-1}\right)\left(1+q^{10 n-2}\right)\left(1-q^{10 n-3}\right)\left(1-q^{10 n-4}\right) \\
& \cdot\left(1-q^{10 n-6}\right)\left(1-q^{10 n-7}\right)\left(1+q^{10 n-8}\right)\left(1+q^{10 n-9}\right) \\
= & \frac{1}{2} \vartheta_{2}(0 \mid 5 \tau) \vartheta_{4}(0 \mid 5 \tau)\left(\frac{\vartheta_{2}}{\vartheta_{4}}\left(\frac{\pi \tau}{2} \mid 5 \tau\right)+\frac{\vartheta_{2}}{\vartheta_{4}}\left(\frac{3 \pi \tau}{2} \mid 5 \tau\right)\right)
\end{aligned}
$$




$$
\begin{aligned}
& \sum_{n=1}^{\infty}(-1)^{n}\left\{\frac{q^{5 n+1}}{1-q^{5 n+1}}-\frac{q^{5 n+2}}{1-q^{5 n+2}}-\frac{q^{5 n+3}}{1-q^{5 n+3}}+\frac{q^{5 n+4}}{1-q^{5 n+4}}\right\} \\
& =\sum_{n=1}^{\infty} \frac{q^{n}-q^{2 n}-q^{3 n}+q^{4 n}}{1+q^{5 n}} \\
& =q \prod_{n=1}^{\infty} \frac{\left(1-q^{5 n}\right)^{2}}{\left(1-q^{10 n-3}\right)\left(1-q^{10 n-4}\right)\left(1-q^{10 n-6}\right)\left(1-q^{10 n-7}\right)} \\
& =\frac{1}{2} \vartheta_{3}\left(0 \mid \frac{5 \tau}{2}\right) \vartheta_{4}\left(0 \mid \frac{5 \tau}{2}\right)\left(\frac{\vartheta_{3}}{\vartheta_{4}}\left(\frac{3 \pi \tau}{4} \mid \frac{5 \tau}{2}\right)-\frac{\vartheta_{3}}{\vartheta_{4}}\left(\frac{\pi \tau}{4} \mid \frac{5 \tau}{2}\right)\right) \text {, } \\
& 1+\sum_{n=1}^{\infty} \frac{q^{n}+q^{2 n}+q^{3 n}+q^{4 n}}{1+q^{5 n}} \\
& =\prod_{n=1}^{\infty} \frac{\left(1-q^{5 n}\right)^{2}}{\left(1-q^{10 n-1}\right)\left(1-q^{10 n-2}\right)\left(1-q^{10 n-8}\right)\left(1-q^{10 n-9}\right)} \\
& =\frac{1}{2} \vartheta_{3}\left(0 \mid \frac{5 \tau}{2}\right) \vartheta_{4}\left(0 \mid \frac{5 \tau}{2}\right)\left(\frac{\vartheta_{3}}{\vartheta_{4}}\left(\frac{\pi \tau}{4} \mid \frac{5 \tau}{2}\right)+\frac{\vartheta_{3}}{\vartheta_{4}}\left(\frac{3 \pi \tau}{4} \mid \frac{5 \tau}{2}\right)\right) \text {, } \\
& \sum_{n=0}^{\infty}(-1)^{n}\left\{\frac{q^{5 n+1}}{1+q^{10 n+2}}+\frac{q^{5 n+2}}{1+q^{10 n+4}}-\frac{q^{5 n+3}}{1+q^{10 n+6}}-\frac{q^{5 n+4}}{1+q^{10 n+8}}\right\} \\
& =q \prod_{n=1}^{\infty}\left(1-q^{n}\right)\left(1+q^{2 n-1}\right)^{2}\left(1-q^{5 n}\right)\left(1+q^{10 n}\right)^{2} \\
& =\frac{1}{2 \sqrt{5}} \vartheta_{3}(0 \mid \tau) \vartheta_{4}(0 \mid \tau)\left(\frac{\vartheta_{3}}{\vartheta_{4}}\left(\frac{\pi}{10} \mid \tau\right)-\frac{\vartheta_{3}}{\vartheta_{4}}\left(\frac{3 \pi}{10} \mid \tau\right)\right) \text {, } \\
& 1+\sum_{n=0}^{\infty}(-1)^{n}\left\{\frac{q^{5 n+1}}{1+q^{10 n+2}}-\frac{q^{5 n+2}}{1+q^{10 n+4}}+\frac{q^{5 n+3}}{1+q^{10 n+6}}-\frac{q^{5 n+4}}{1+q^{10 n+8}}\right\} \\
& =\prod_{n=1}^{\infty}\left(1-q^{n}\right)^{2}\left(1+q^{n}\right)^{3}\left(1-q^{10 n-5}\right) \\
& =\frac{1}{2} \vartheta_{3}(0 \mid \tau) \vartheta_{4}(0 \mid \tau)\left(\frac{\vartheta_{3}}{\vartheta_{4}}\left(\frac{3 \pi}{10} \mid \tau\right)+\frac{\vartheta_{3}}{\vartheta_{4}}\left(\frac{\pi}{10} \mid \tau\right)\right) \text {, } \\
& \sum_{n=1}^{\infty} \frac{n\left(q^{n}-q^{2 n}-q^{3 n}+q^{4 n}\right)}{1-q^{5 n}} \\
& =q \prod_{n=1}^{\infty} \frac{\left(1-q^{5 n}\right)^{5}}{1-q^{n}}=\frac{1}{4}\left(\frac{\vartheta_{4}^{5}(0 \mid 5 \tau)}{\vartheta_{4}(0 \mid \tau)}-\vartheta_{4}(0 \mid \tau) \vartheta_{4}^{3}(0 \mid 5 \tau)\right) \\
& =\frac{1}{4}\left\{\left(\frac{\vartheta_{4}^{\prime}}{\vartheta_{4}}\right)^{\prime}\left(\frac{3 \pi \tau}{4} \mid \frac{5 \tau}{2}\right)-\left(\frac{\vartheta_{4}^{\prime}}{\vartheta_{4}}\right)^{\prime}\left(\frac{\pi \tau}{4} \mid \frac{5 \tau}{2}\right)\right\} \text {, }
\end{aligned}
$$




$$
\begin{aligned}
& \sum_{n=0}^{\infty}(-1)^{n}\left\{\frac{(5 n+1) q^{5 n+1}}{1-q^{10 n+2}}+\frac{(5 n+2) q^{5 n+2}}{1-q^{10 n+4}}-\frac{(5 n+3) q^{5 n+3}}{1-q^{10 n+6}}-\frac{(5 n+4) q^{5 n+4}}{1-q^{10 n+8}}\right\} \\
& =q \prod_{n=1}^{\infty}\left(1+q^{2 n-1}\right)^{2}\left(1-q^{2 n}\right)^{3}\left(1-q^{5 n}\right)\left(1-q^{10 n-5}\right)\left(1+q^{10 n}\right)^{2} \\
& \quad=\frac{1}{4 \sqrt{5}}\left\{\left(\frac{\vartheta_{4}^{\prime}}{\vartheta_{4}}\right)^{\prime}\left(\frac{\pi}{10} \mid \tau\right)-\left(\frac{\vartheta_{4}^{\prime}}{\vartheta_{4}}\right)^{\prime}\left(\frac{3 \pi}{10} \mid \tau\right)\right\} .
\end{aligned}
$$

\section{Partition identities}

We will single out three identities (3.1), (3.39), and (3.40) for discussions.

First, using an observation originally due to Bailey and communicates to the author by Professor F. G. Garvan, we see that (3.1) yields the following identity:

$$
\sum_{n=0}^{\infty} p^{d, o}(5 n+4) q^{n}=\prod_{n=1}^{\infty} \frac{\left(1-q^{5 n}\right)^{2}\left(1+q^{10 n}\right)^{2}\left(1+q^{10 n-5}\right)}{\left(1-q^{n}\right)^{2}\left(1+q^{2 n}\right)^{2}}
$$

where $p^{d, o}(n)=$ the number of ways an integer $n$ can be written as a sum of distinct odd positive integers.

The proof goes as follows: In (3.1), we write

$$
\begin{aligned}
q \prod_{n=1}^{\infty} & \left(1+q^{2 n-1}\right)\left(1+q^{5 n}\right)\left(1-q^{5 n}\right)^{2}\left(1+q^{10 n}\right) \\
& =\prod_{n=1}^{\infty}\left(1+q^{5 n}\right)\left(1-q^{5 n}\right)^{2}\left(1+q^{10 n}\right) \sum_{n=0}^{\infty} p^{d, o}(n) q^{n+1} \\
& =\prod_{n=1}^{\infty}\left(1+q^{5 n}\right)\left(1-q^{5 n}\right)^{2}\left(1+q^{10 n}\right) \sum_{m=0}^{4} \sum_{n=0}^{\infty} p^{d, o}(5 n+m) q^{5 n+m+1}
\end{aligned}
$$

and

$$
\sum_{n=1}^{\infty} \frac{q^{n}-q^{3 n}-q^{7 n}+q^{9 n}}{1+q^{10 n}}=\sum_{m=0}^{4} \sum_{n=0}^{\infty} A_{5 n+m} q^{5 n+m}, \quad A_{o}=0 .
$$

We now sum over only the terms associated with $q^{5 n}, n=0,1,2, \ldots$, of the above two power series. It is elementary to see that

$$
\begin{aligned}
\prod_{n=0}^{\infty}\left(1+q^{5 n}\right)\left(1-q^{5 n}\right)^{2}\left(1+q^{10 n}\right) \sum_{n=0}^{\infty} p^{d, o}(5 n+4) q^{5 n+5} \\
\quad=\sum_{n=0}^{\infty} A_{5 n} q^{5 n}=\sum_{n=1}^{\infty} \frac{q^{5 n}-q^{15 n}-q^{35 n}+q^{45 n}}{1+q^{50 n}} \\
=q^{5} \prod_{n=1}^{\infty}\left(1+q^{10 n-5}\right)\left(1+q^{25 n}\right)\left(1+q^{25 n}\right)^{2}\left(1+q^{50 n}\right)
\end{aligned}
$$

From the first part and the last part of the above identity, we obtain the desired result after replacing $q^{5}$ by $q$. 
Similarly, following the identical argument as above, one sees that the identities (3.39) and (3.40) yield, respectively,

(4.2)

$$
\begin{aligned}
& \sum_{n=0}^{\infty} p(5 n+4 ; 3,4,6,7) q^{n} \\
& \quad=\prod_{n=1}^{\infty} \frac{\left(1-q^{5 n}\right)^{2}}{\left(1-q^{n}\right)^{2}} \cdot \frac{1}{\left(1-q^{10 n-3}\right)\left(1-q^{10 n-4}\right)\left(1-q^{10 n-6}\right)\left(1-q^{10 n-7}\right)}, \\
& \quad \sum_{n=0}^{\infty} p(5 n ; 1,2,8,9) q^{n} \\
& \quad=\prod_{n=1}^{\infty} \frac{\left(1-q^{5 n}\right)^{2}}{\left(1-q^{n}\right)^{2}} \frac{1}{\left(1-q^{10 n-1}\right)\left(1-q^{10 n-2}\right)\left(1-q^{10 n-8}\right)\left(1-q^{10 n-9}\right)},
\end{aligned}
$$

where $p(n ; a, b, c, d)$ denotes the number of ways an integer $n$ can be written as a sum of positive integers $\equiv a, b, c, d(\bmod 10)$.

We note that $(3.39) \times(3.40)=(3.43)$ and (3.43) yields one of the most celebrated identities (1.14) of Ramanujan which we have mentioned earlier. And it is interesting to see that the product of (4.2) and (4.3) is

$$
\left(\sum_{n=0}^{\infty} p(5 n+4 ; 3,4,6,7) q^{n}\right)\left(\sum_{n=0}^{\infty} p(5 n ; 1,2,8,9) q^{n}\right)=\prod_{n=1}^{\infty} \frac{\left(1-q^{5 n}\right)^{5}}{\left(1-q^{n}\right)^{5}}
$$

which "almost" equals (1.14).

\section{CONCLUDING REMARKS}

First, we give a brief comments on the identity (1.1). This identity follows immediately from the general identity

$$
\begin{aligned}
\vartheta_{1}(u-b) & \vartheta_{1}(a-s) \vartheta_{1}(u-s+y) \vartheta_{1}(a-b+y) \\
& -\vartheta_{1}(u-s) \vartheta_{1}(a-b) \vartheta_{1}(u-b+y) \vartheta_{1}(a-s+y) \\
= & \vartheta_{1}(y) \vartheta_{1}(u-a) \vartheta_{1}(b-s) \vartheta_{1}(u-b+a-s+y)
\end{aligned}
$$

by choosing $y=\pi / 2$ and letting $x=u-b, y=s-u$, and $z=a-s$. It is worthwhile to note that there is also a version of $(5.1)$ for the theta functions on the Riemann surfaces. It is the so-called "the trisecant identity of Fay" (see $[2$, p. $34,(45)]$ ) which plays a vital role in the study of Riemann surfaces; when the genus of the Riemann surface is 1 , it reduces to (5.1). It will be interesting to see if any of the identities discussed in this work can be formulated in terms of the theta functions for the Riemann surfaces.

In a recent work [4], the identity (5.1) is also used to study the properties of the Green's function and the Szegö kernel for an annulus and, as a consequence, several extremal problems are solved. And most surprisingly, (5.1) and its generalization for multiply connected domains provided a very elegant proof to the Nevanlinna-Pick interpolation problem in [3].

The proof of (5.1) is very elementary. It goes as follows: Let $F(u, a, b, s)$ be the ratio of the quantities on both sides of the identity (5.1) with the numerator the difference of the four theta products and the denominator the product of the four theta functions. We will show $F \equiv 1$. To this end, we first fix $a, b$, 
and $s$ and consider $F$ as a function of $u$. First, we show that $F$ is entire. Since $F$ is doubly periodic, it suffices to show that $F$ is analytic at $u=a$ and $u=b-a+s-y$ which are the locations of the possible poles of $F$. We observe that at $u=a$ and $u=b-a+s-y$, the numerator of $F$ is equal to zero and since the poles of $F$ are all of multiplicity one, $F$ is analytic at $u=a$ and $u=b-a+s-y$. Therefore $F$ is analytic in the entire complex plane. From the fact that a doubly periodic entire function is constant, we conclude that $F$ is independent of $u$. Repeating the same argument for the variables $a, b$, and $s$, we conclude that $F$ is independent of $u, a, b$, and $s$, so it is a constant. Now let $a=s$, we see that this constant is 1 . This establishes (5.1).

We conclude the paper by bring the reader's attention to Chapter 19 of the recent book Ramanujan's Notebook. III by Professor Berndt [1] in which many identities of this paper are established using the notation as well as the methods which Ramanujan might have employed. It is, however, the opinion of this author that the traditional notation of theta functions and their well-known properties seem to provide greater clarity as well as simplicity and cohesion to this subject.

\section{REFERENCES}

1. B.C. Berndt, Ramanujan's notebook. III, Springer-Verlag, New York, 1991.

2. J.D. Fay, Theta functions on Riemann surfaces, Springer-Verlag, New York, 1973.

3. S.A. McCullough, Nevanlinna-Pick interpolation and canonical mapping functions, preprint.

4. S.A. McCullough and Li-Chien Shen, On the Szegö kernel of an annulus, Proc. Amer. Math. Soc. (to appear).

5. E.T. Whittaker and G.N. Watson, Modern analysis, 4th ed., Cambridge Univ. Press, London and New York, 1958.

Department of Mathematics, University of Florida, Gainesville, Florida 32611-2082

E-mail address: shen@math.ufl.edu. 